\title{
A GALERKIN-PETROV METHOD FOR SINGULAR INTEGRAL EQUATIONS
}

\author{
DAVID ELLIOTT'
}

(Received 28 April 1982; revised 5 October 1982)

\begin{abstract}
A Galerkin-Petrov method for the approximate solution of the complete singular integral equation with Cauchy kernel, based upon the use of two sets of orthogonal polynomials, is considered. The principal result of this paper proves convergence of the approximate solutions to the exact solution making use of a convergence theorem previously given by the author. In conclusion, some related topics such as a first iterate of the approximate solution and a discretized Galerkin-Petrov method are considered. The paper extends to a much more general equation many results obtained by other authors in particular cases.
\end{abstract}

\section{Introduction}

The singular integral equation to be considered in this paper is that defined on $-1<t<1$ by

$$
a(t) \phi(t)+\frac{b(t)}{\pi} f_{-1}^{1} \frac{\phi(\tau) d \tau}{\tau-t}+\int_{-1}^{1} k(t, \tau) \phi(\tau) d \tau=y(t)
$$

where the Cauchy principal value integral $f_{-1}^{1}(\phi(\tau) /(\tau-t)) d \tau$ is defined in the usual way by

$$
\lim _{\varepsilon \rightarrow 0+}\left(\int_{-1}^{t-\varepsilon}+\int_{t+\varepsilon}^{1}\right) \frac{\phi(\tau) d \tau}{\tau-t}
$$

The real functions $a, b, k$ and $y$ appearing in (1.1) are given and we shall require approximate solutions to the unknown function $\phi$. Following Muskhelishvili [17], we shall assume that $a$ and $y$ are Hölder continuous on $[-1,1]$ and that $k$ is Hölder continuous on $[-1,1] \times[-1,1]$. We shall assume throughout this paper

\footnotetext{
'Mathematics Department, University of Tasmania, Box 252C, G.P.O., Hobart, Tasmania 7001.

(c) Copyright Australian Mathematical Society 1983, Serial-fee code 0334-2700/83.
} 
that $b$ is a polynomial of degree $\mu$. We shall look for solutions $\phi$ of (1.1) which are Hölder continuous on all closed intervals interior to $(-1,1)$ and integrable at the end points \pm 1 . In Muskhelishvili's terminology $[17$, Sections 79,107$]$ we shall look for solutions $\phi$ in the class of functions $h_{0}$ which is the widest class of all possible solutions. Relative to $h_{0}$, we shall assume that the index of (1.l) is given by $\kappa$, where $\kappa$ is an integer which may be positive, negative or zero. When $\kappa>0$, we shall require an extra $\kappa$ condition on (1.1) to make the solution unique; when $\kappa<\overline{0}$, the function $y$ and solution $\phi$ will be required to satisfy $(-\kappa)$ consistency conditions. Only when $\kappa=0$ does (1.1) have a unique solution.

In this paper we shall consider a Galerkin-Petrov method (see Krasnosel'skii $e t$ al. [13, Chapter 4]) for the approximate solution of (1.1). The method is based upon the use of two sets of polynomials which are orthogonal with respect to non-negative, integrable weight functions defined on the interval $(-1,1)$. This gives rise to a global approximation method which contrasts with the local approximation Galerkin method discussed by Thomas [21] for the particular case of (1.1) with $a=1, b$ smooth and $\kappa=0$.

The Galerkin-Petrov method of this paper has been discussed previously by many authors for particular cases of (1.1). Thus the case when $a=0, b=1$ has been discussed by Erdogan [6], Erdogan and Gupta [5], Fromme and Golberg [7; Chapters 4 and 5] and Linz [16]. The slightly more general case when $a, b$ are arbitrary constants has been considered by Karpenko [12], Dzhishkariani [1], Ioakimidis [10], Krenk [14] and Golberg [8]. It is relevant to note that Junghanns and Silbermann [11] have recently given an abstract theory for the convergence of approximate solutions of (1.1) although the particular application of the theory in [11] is concerned with collocation methods based on the use of quadrature formulae for the integrals in (1.1).

For the generality of (1.1) to be discussed in this paper we shall first of all outline the method which was previously described by the author in [7; Chapter 3]. The main purpose of this paper is to discuss the convergence of the method using the general convergence theorem given by the author in [2]. This has previously been used [3] to demonstrate the convergence of the method of classical collocation; here we shall show, in Section 3, that the theorem can be applied as well in this context. In Section 4 we shall comment briefly on three associated topics. These are: (i) the equivalence of direct and indirect methods of solution of (1.1); (ii) an iterated solution which converges more quickly than the original approximate solution; and (iii) a "discretized" Galerkin-Petrov method where inner products are replaced by certain quadrature sums so that we recover the collocation solution of [3].

Before we embark upon a description of the Galerkin-Petrov method we shall require some preliminary results from the theory of singular integral equations. 
As is usual when solving (1.1) we shall not solve directly for $\phi$ but for a closely related function $\psi$ where

$$
\phi=Z \psi / r \text {. }
$$

Here $Z$ denotes the fundamental function of (1.1) for the solution in the class $h_{0}$; $r=\left(a^{2}+b^{2}\right)^{1 / 2}$, it being assumed throughout that $r(t)>0$ for all $t \in[-1,1]$. It turns out (see, for example, [4]) that on $[-1,1]$ both $Z$ and $1 / Z$ are non-negative and integrable so that we may introduce on $(-1,1)$ two non-negative, integrable weight functions $w_{1}$ and $w_{2}$ say, defined by

$$
w_{1}=Z / r, \quad w_{2}=1 / Z r .
$$

If we introduce Tricomi's notation [22] where

$$
\sigma(\phi ; t)=\frac{1}{\pi} f_{-1}^{1} \frac{\phi(\tau) d \tau}{\tau-t},
$$

then from (1.2) and (1.3) we can rewrite (1.1) as

$$
A \psi+K \psi=y,
$$

where

$$
\left.\begin{array}{l}
A \psi=a w_{1} \psi+b \mathcal{J}\left(w_{1} \psi\right), \\
K \psi=\int_{-1}^{1} w_{1}(\tau) k(t, \tau) \psi(\tau) d \tau .
\end{array}\right\}
$$

In [4] we have discussed in some detail the orthogonal polynomials which are induced by the weight functions $w_{1}$ and $w_{2}$. In this paper we shall let $\left\{t_{n}\right\},\left\{u_{n}\right\}$ denote the sequences of orthonormal polynomials induced by the weight functions $w_{1}, w_{2}$ respectively where $t_{n}, u_{n}$ are polynomials of exact degree $n$; the coefficient of their highest power always being positive. Thus we shall have

$$
\int_{-1}^{1} w_{1}(\tau) t_{j}(\tau) t_{k}(\tau) d \tau=\delta_{j, k}
$$

and

$$
\int_{-1}^{1} w_{2}(\tau) u_{j}(\tau) u_{k}(\tau) d \tau=\delta_{j, k}
$$

for $j, k=0,1,2, \ldots$. Let $H_{i}, i=1,2$, denote real separable Hilbert spaces with inner products defined by

$$
\left(\psi_{1}, \psi_{2}\right)_{H_{s}}=\int_{-1}^{1} w_{1}(\tau) \psi_{1}(\tau) \psi_{2}(\tau) d \tau .
$$

Then the polynomials $\left\{t_{n}\right\},\left\{u_{n}\right\}$ can be taken as bases for $H_{1}, H_{2}$ respectively. As shown in [4] we can look upon both $A$ and $K$ as bounded linear operators on $H_{1}$ into $\mathrm{H}_{2}$. 
Finally, we recall some properties of the operator $A$ and some closely related operators. For further discussions on these the reader is referred to [3] and [4]. Since we suppose $A$ has index $\kappa$ then $\operatorname{dim} \operatorname{ker}(A)$, the dimension of the null space of $A$, is given by $\max \{0, \kappa\}$. When $\kappa \geqslant 1$, we have

$$
\operatorname{ker}(A)=\operatorname{span}\left\{b t_{0}, b t_{1}, \ldots, b t_{\kappa-1}\right\} .
$$

It is not difficult to show that rant $A)$ is orthogonal to the space spanned by $\left\{1, t, \ldots, t^{-\kappa-1}\right\}$, this set being other than the zero element when $\kappa \leqslant-1$. In other words

$$
\left(u_{k-1}, A \psi\right)_{H_{2}}=0, \quad k=1(1)(-\kappa),
$$

for all $\psi \in \operatorname{dom}(A)$. Next, we introduce the adjoint of $A$, which we denote by $A^{*}$, this being such that for all $\psi_{1}, \psi_{2}$ we have

$$
\left(A \psi_{1}, \psi_{2}\right)_{H_{2}}=\left(\psi_{1}, A^{*} \psi_{2}\right)_{H_{1}} \text {. }
$$

(Note that this definition is different from that given in Section 2 of [2].) From (1.12) it is straightforward to show that

$$
A^{*} \psi=a w_{2} \psi-\mathcal{T}\left(b w_{2} \psi\right)
$$

Now $A^{*}$ is an operator from $H_{2}$ into $H_{1}$; a closely related operator is $\hat{A}^{I}$ which is defined by

$$
\hat{A}^{\prime} \psi=a w_{2} \psi-b \mathcal{T}\left(w_{2} \psi\right)
$$

and has the property that

$$
\left.\begin{array}{l}
A \hat{A}^{I} \psi=\psi, \quad \text { for all } \psi, \text { when } \kappa \geqslant 0, \\
\hat{A}^{I} A \psi=\psi, \text { for all } \psi, \text { when } \kappa \leqslant 0 .
\end{array}\right\}
$$

Thus $\hat{A}^{\prime}$ is a right inverse of $A$ when $\kappa \geqslant 0$ and a left inverse when $\kappa \leqslant 0$. Only when $\kappa=0$ is $\hat{A}^{I}$ the inverse of $A$. We also observe that $\hat{A}^{I}=A^{*}$ only when $b$ is a constant. With these operators we can now give some relationships between the polynomials $t_{n}$ and $u_{n}$. If $m=n-\kappa$ then, from [4], we have

$$
\begin{array}{ll}
\text { for } n \geqslant \max (\mu, \kappa), & A t_{n}=(-1)^{\kappa} u_{m}, \\
\text { for } n \geqslant \max (\mu, \kappa), & \hat{A}^{\prime} u_{m}=(-1)^{\kappa} t_{n}, \\
\text { for } n \geqslant \max (0, \kappa), & A^{*} u_{m}=(-1)^{\kappa} t_{n} .
\end{array}
$$




\section{A Galerkin-Petrov method}

To any arbitrary positive integer $n$, we seek an approximate solution, $\psi_{n}$ say, of (1.5) in the form

$$
\psi_{n}=\sum_{j=1}^{n} \xi_{j} t_{j-1} .
$$

Substituting (2.1) into (1.5) gives a residual $R_{n}$ say where

$$
R_{n}=(A+K) \psi_{n}-y
$$

and for the Galerkin-Petrov method we choose the constants $\xi_{1}, \xi_{2}, \ldots, \xi_{n}$ in (2.1) such that

$$
\left(u_{i-1}, R_{n}\right)_{H_{2}}=0, \quad i=1(1) m,
$$

where $m=n-\kappa$. This gives rise to a system of $m$ equations in $n$ unknowns which we can write as

$$
\left(A_{n}+K_{n}\right) x_{n}=y_{m} \text {, }
$$

where $A_{n}, K_{n}$ are $m \times n$ matrices, $x_{n}$ is the $n \times 1$ column vector $\left(\xi_{1}, \xi_{2}, \ldots, \xi_{n}\right)^{T}$ and $y_{m}$ is the $m \times 1$ column vector $\left(\left(u_{0}, y\right)_{H_{2}},\left(u_{1}, y\right)_{H_{2}}, \ldots,\left(u_{m-1}, y\right)_{H_{2}}\right)^{T}$. (For a discussion as to why we require $m$ equations in $n$ unknowns, see [2].) From (2.3) if we write $A_{n}=\left(a_{i, j}^{(n)}\right)$ and $K_{n}=\left(k_{i, j}^{(n)}\right)$ then we have

$$
a_{i, j}^{(n)}=\left(u_{t-1}, A t_{j-1}\right)_{H_{2}}, \quad k_{i, j}^{(n)}=\left(u_{t-1}, K t_{j-1}\right)_{H_{2}},
$$

for $i=1(1) m, j=1(1) n$. Now, from (1.16), we see that for $i=1(1) m$ and $j \geqslant 1+\max (\mu, \kappa)$

$$
a_{i, j}^{(n)}=(-1)^{\kappa} \delta_{i-1, j-1-\kappa},
$$

so that much of the matrix $A_{n}$ has a simple structure. Again, we might note from (1.11) that when $\kappa \leqslant-1$, the first $(-\kappa)$ rows of $A_{n}$ are zero so that (2.4) is not solvable for arbitrary $y_{m}$, but we must have that the so called consistency conditions are satisfied. These are that $y_{m}-K_{n} x_{n}$ is orthogonal to $\operatorname{ker}\left(A_{n}^{T}\right)$.

Before discussing convergence we shall now introduce appropriate restriction and prolongation operators so that we can relate equations (1.5) and (2.4). (For further discussion on these operators see Noble [18], Linz [15] and Thomas [20].) If $X_{n}$ denotes the space of $n \times 1$ column vectors we define

$$
\left.\begin{array}{l}
r_{n}: H_{1} \rightarrow X_{n} \text { such that } r_{n} x=\left(\left(t_{0}, x\right)_{H_{1}}, \ldots,\left(t_{n-1}, x\right)_{H_{1}}\right)^{T}, \\
p_{n}: X_{n} \rightarrow H_{1} \quad \text { such that } p_{n} x_{n}=\sum_{i=1}^{n} \xi_{i} t_{t-1}, x_{n}=\left(\xi_{1}, \xi_{2}, \ldots, \xi_{n}\right)^{T} .
\end{array}\right\}
$$

We observe that $r_{n} p_{n}=I_{n}$. If we take the norm in $X_{n}$ to be the 2-norm so that

$$
\left\|x_{n}\right\|_{X_{n}}=\left\|x_{n}\right\|_{2}=\left\|p_{n} x_{n}\right\|_{H_{1}} \text {, }
$$


we can define $\left\|r_{n}\right\|$ by

$$
\left\|r_{n}\right\|=\sup _{x \neq 0}\left\{\left\|r_{n} x\right\|_{X_{n}} /\|x\|_{H_{1}}\right\}
$$

and it is straightforward to show that $\left\|r_{n}\right\|=1$. Again, if we define

$$
\left\|p_{n}\right\|=\sup _{x_{n} \neq 0}\left\{\left\|p_{n} x_{n}\right\|_{H_{1}} /\left\|x_{n}\right\|_{X_{n}}\right\}
$$

then we have $\left\|p_{n}\right\|-1$. We procecd similarly with iesiriciion and proiongation operators between the spaces $H_{2}$ and $Y_{m}$ say, the space of all $m \times 1$ column vectors. We have

$$
\left.\begin{array}{l}
s_{m}: H_{2} \rightarrow Y_{m} \text { such that } s_{m} y=\left(\left(u_{0}, y\right)_{H_{2}}, \ldots,\left(u_{m-1}, y\right)_{H_{2}}\right)^{T}, \\
q_{m}: Y_{m} \rightarrow H_{2} \quad \text { such that } q_{m} y_{m}=\sum_{j=1}^{m} \eta_{j} u_{j-1},
\end{array}\right\}
$$

where $y_{m}=\left(\eta_{1}, \eta_{2}, \ldots, \eta_{m}\right)^{T}$, so that $s_{m} q_{m}=I_{m}$. Again if we define

$$
\left\|y_{m}\right\|_{Y_{m}}=\left\|y_{m}\right\|_{2}=\left\|q_{m} y_{m}\right\|_{H_{2}} \text {, }
$$

then it follows that $\left\|s_{m}\right\|=\left\|q_{m}\right\|=1$.

Let us return now to the Galerkin-Petrov method; we see that in terms of the above operators, we obtain (2.4) as

$$
s_{m}(A+K) p_{n} x_{n}=s_{m} y,
$$

so that the $A_{n}, K_{n}$ are given by

$$
A_{n}=s_{m} A p_{n}, \quad K_{n}=s_{m} K p_{n} .
$$

It is apparent from the definitions of the preceding paragraph that both $p_{n} r_{n}$ and $q_{m} s_{m}$ are projections. In particular if we let $T_{n}=\operatorname{span}\left\{t_{0}, t_{1}, \ldots, t_{n-1}\right\}$ and if $P_{n}$ denotes the orthogonal projection from $H_{1}$ onto $T_{n}$, then $P_{n}=p_{n} r_{n}$ with $\left\|P_{n}\right\|=1$. Similarly, if we let $U_{n}=\operatorname{span}\left\{u_{0}, u_{1}, \ldots, u_{n-1}\right\}$ then $Q_{n}$, the orthogonal projection from $\mathrm{H}_{2}$ onto $U_{n}$, is given by $Q_{n}=q_{n} s_{n}$ and $\left\|Q_{n}\right\|=1$. The following lemma is important for our subsequent analysis.

LEMMA 2.1. For $n \geqslant 1+\max (\mu, \kappa)$,

$$
A P_{n}=Q_{m} A, \quad P_{n} \hat{A}^{I}=\hat{A}^{I} Q_{m} .
$$

Proof. This depends on $(1.16)-(1.18)$. Let us consider the first of (2.14). Since $x \sim \sum_{i=1}^{\infty}\left(t_{i-1}, x\right)_{H_{1}} t_{i-1}$ then

$$
A\left(I-P_{n}\right) x=A \sum_{i=n}^{\infty}\left(t_{t-1}, x\right)_{H_{1} t_{i-1}}=(-1)^{\kappa} \sum_{i=n}^{\infty}\left(t_{t-1}, x\right)_{H_{1} u_{t-\kappa-1}} \text {, }
$$


by (1.16) since $n-1 \geqslant \max (\mu, \kappa)$. Again

$$
\begin{aligned}
\left(I-Q_{m}\right) A x & =\left(I-Q_{m}\right) \sum_{j=1}^{\infty}\left(u_{j-1}, A x\right)_{H_{2}} u_{j-1} \\
& =\sum_{j=m}^{\infty}\left(u_{j-1}, A x\right)_{H_{2}} u_{j-1}=\sum_{j=m}^{\infty}\left(A^{*} u_{j-1}, x\right)_{H_{1}} u_{j-1} \\
& =(-1)^{\kappa} \sum_{j=m}^{\infty}\left(t_{j-1+\kappa}, x\right)_{H_{1}} u_{j-1}, \text { by }(1.18) \\
& =(-1)^{\kappa} \sum_{i=n}^{\infty}\left(t_{t-1}, x\right)_{H_{1}} u_{t-\kappa-1}=A\left(I-P_{n}\right) x,
\end{aligned}
$$

from above. Thus $\left(I-Q_{m}\right) A=A\left(I-P_{n}\right)$ for $n \geqslant 1+\max (\mu, \kappa)$ and the result follows. The second part of (2.14) follows similarly.

The first of (2.13) defines the $m \times n$ matrix $A_{n}$. We would like to show that this matrix is of rank given by $\min (m, n)$ and, recalling (1.15), this will follow if we can show that for $\kappa \geqslant 0, A_{n}$ possesses a right inverse and for $\kappa \leqslant 0$ that it possesses a left inverse. Let us then define an $n \times m$ matrix $\hat{A}_{m}^{I}$ by

$$
\hat{A}_{m}^{I}=r_{n} \hat{A}^{I} q_{m} \text {, }
$$

and show that this matrix is the required inverse for all values of $\kappa$.

THEOREM 2.2. For $n \geqslant 1+\max (\mu, \kappa)$,

$$
\left.\begin{array}{l}
A_{n} \hat{A}_{m}^{\prime}=I_{m}, \quad \text { when } \kappa \geqslant 0, \\
\hat{A}_{m}^{\prime} A_{n}=I_{n}, \quad \text { when } \kappa \leqslant 0 .
\end{array}\right\}
$$

Proof. Suppose that $\kappa \geqslant 0$. Then

$$
\begin{aligned}
A_{n} \hat{A}_{m}^{\prime} & =\left(s_{m} A p_{n}\right)\left(r_{n} \hat{A}^{\prime} q_{m}\right)=s_{m}\left(A P_{n}\right) \hat{A}^{I} q_{m} \\
& =s_{m}\left(Q_{m} A\right) \hat{A}^{\prime} q_{m}, \quad \text { by }(2.14), \\
& =s_{m}\left(q_{m} s_{m}\right)\left(A \hat{A}^{I}\right) q_{m} \\
& =\left(s_{m} q_{m}\right) s_{m} I q_{m}, \quad \text { by }(1.15) \text { since } \kappa \geqslant 0, \\
& =I_{m}, \quad \text { since } s_{m} q_{m}=I_{m} .
\end{aligned}
$$

The proof of the second part of (2.16) for $\kappa \leqslant 0$ follows similarly.

Thus we have identified the appropriate inverses and it follows that $A_{n}$ is of rank $=\min (m, n)$. With these preliminaries established we may now consider the problem of convergence. 


\section{Convergence of the approximate solutions}

We shall consider the convergence theorem for singular integral equations as given by the author in [2; Theorem 5.5] and shall show that all the conditions of that theorem are satisfied by the Galerkin-Petrov method described in the previous section. It turns out that that theorem is slightly more general than we shall need here since $\left\{A_{n}\right\}$ is not only $\alpha$-stable but stable. That is (see (iii) below) we have that $\left\|\hat{A}_{m}^{i}\right\| \leqslant C_{1}$, for all $m$ so that in considering the conditions of Theorem 5.5 of [2] we can throughout suppress the prefix " $\alpha$-." Let us now consider conditions (i) to (vii) of that theorem in turn.

(i) $\left\{A_{n}\right\}$ is compatible with $A$. In other words, we need to show that $\operatorname{ker}\left(A_{n}\right)=$ $r_{n}\{\operatorname{ker}(A)\}$. This result is, of course, trivially true when $\kappa \leqslant 0$ since the null spaces then consists only of the zero element. Suppose $\kappa \geqslant 1$, then from $(1.10) \operatorname{ker}(A)$ is of dimension $\kappa$. If $x$ is any element out of $\operatorname{ker}(A)$ then

$$
\begin{aligned}
A_{n}\left(r_{n} x\right) & =\left(s_{m} A p_{n}\right) r_{n} x, \quad \text { by }(2.13), \\
& =s_{m} A P_{n} x \\
& =\left(s_{m} Q_{m}\right)(A x), \quad \text { by Lemma } 2.1, \\
& =0 .
\end{aligned}
$$

Thus $r_{n} x \in \operatorname{ker}\left(A_{n}\right)$. Arguing similarly we can show that if $A_{n} x_{n}=0$ then $A\left(p_{n} x_{n}\right)=0$ so that we have a one-to-one correspondence between the null spaces of $A_{n}$ and $A$. Compatibility now follows.

(ii) $\left\{A_{n}\right\}$ is discretely consistent with $A$ on $\operatorname{dom}(A)$. We have to show that for all $x \in \operatorname{dom}(A)$, the sequence $\left\{\delta_{n}^{A} x\right\}$ is null where

$$
\delta_{n}^{A} x=s_{m} A x-A_{n} r_{n} x \text {. }
$$

From (2.13) we have that

$$
\begin{aligned}
\delta_{n}^{A} x & =s_{m} A x-s_{m} A P_{n} x \\
& =s_{m} A x-\left(s_{m} Q_{m}\right) A x, \quad \text { by Lemma } 2.1, \\
& =0, \quad \text { since } s_{m} q_{m}=I_{m} .
\end{aligned}
$$

Thus for all $n \geqslant 1+\max (\mu, \kappa)$ we have that $\delta_{n}^{A} x=0$, so that the sequence $\left\{\delta_{n}^{A} x\right\}$ is certainly null.

(iii) $\left\{A_{n}\right\}$ is stable. If we define the norm of $\hat{A}_{m}^{\prime}$ by

$$
\left\|\hat{A}_{m}^{I}\right\|=\sup _{y_{m} \neq 0}\left\{\left\|\hat{A}_{m}^{I} y_{m}\right\|_{X_{n}} /\left\|y_{m}\right\|_{Y_{m}}\right\}
$$

then to show that $\left\{A_{n}\right\}$ is stable we need to show that $\left\|\hat{A}_{m}^{\prime}\right\|$ is uniformly bounded for all $m$ large enough. Now the norm of $\hat{A}^{I}$ is defined by

$$
\left\|\hat{A}^{I}\right\|=\sup _{y \neq 0}\left\{\left\|\hat{A}^{I} y\right\|_{H_{1}} /\|y\|_{H_{2}}\right\}
$$


and arguing as in [4, Section 5] it can be shown that $\hat{A}^{I}$ is a bounded linear operator from $H_{2}$ into $H_{1}$. We have

$$
\begin{aligned}
\left\|\hat{A}_{m}^{I} y_{m}\right\|_{X_{n}} & =\left\|p_{n} \hat{A}_{m}^{I} y_{m}\right\|_{H_{1}}, \quad \text { by }(2.8), \\
& =\left\|P_{n} \hat{A}^{I} q_{m} y_{m}\right\|_{H_{1}}, \quad \text { by }(2.15), \\
& \leqslant\left\|P_{n}\right\| \cdot\left\|\hat{A}^{I}\right\| \cdot\left\|q_{m} y_{m}\right\|_{H_{2}}, \quad \text { by }(3.3), \\
& =\left\|\hat{A}^{I}\right\| \cdot\left\|y_{m}\right\|_{Y_{m}}, \quad \text { by }(2.12) .
\end{aligned}
$$

It follows that $\left\|\hat{A}_{m}^{I}\right\| \leqslant\left\|\hat{A}^{\prime}\right\|$, and, since the right hand side is independent of $m$, the result follows.

(iv) $\operatorname{ran}\left(K_{n}\right) \subseteq \operatorname{ran}\left(A_{n}\right)$. This condition, taken together with $y_{m} \in \operatorname{ran}\left(A_{n}\right)$ will ensure that the algebraic equations $\left(A_{n}+K_{n}\right) x_{n}=y_{m}$ are consistent for $n$ large enough. This is of particular relevance when $\kappa<0$ since it is not a priori evident that $y_{m}-K_{n} x_{n}$ will be orthogonal to $\operatorname{ker}\left(A_{n}\right)$. These conditions may be checked $a$ posteriori and we shall assume that they are satisfied.

(v) $\left\{K_{n}\right\}$ is discretely consistent with $K$ on $X^{(2)} \supseteq \operatorname{dom}(A)$. If we define

$$
\delta_{n}^{K} x=s_{m} K x-K_{n} r_{n} x,
$$

then we need to show that for all $x$, which are in $\operatorname{dom}(A)$ at least, then $\left\{\delta_{n}^{K} x\right\}$ is null. Since we have assumed that $k$ is Hölder continuous on $[-1,1] \times[-1,1]$, it follows that $K$ is compact, From the definition of $K_{n}$ as given by (2.13), and since $P_{n}=p_{n} r_{n}$, we have that $\delta_{n}^{K} x=s_{m} K\left(I-P_{n}\right) x$. Now

$$
\begin{aligned}
\left\|\delta_{n}^{K} x\right\|_{Y_{m}} & =\left\|q_{m} \delta_{n}^{K} x\right\|_{H_{2}}, \quad \text { by }(2.12), \\
& =\left\|Q_{m} K\left(I-P_{n}\right) x\right\|_{H_{2}} \\
& \leqslant\left\|K\left(I-P_{n}\right)\right\| \cdot\|x\|_{H_{1}}, \quad \text { since }\left\|Q_{m}\right\|=1 .
\end{aligned}
$$

If we write $\alpha_{n}=\left\|K\left(I-P_{n}\right)\right\|$ then we know that $\lim _{n \rightarrow \infty} \alpha_{n}=0$, since $K$ is compact (see, for example, [19]). Hence $\left\|\delta_{n}^{K} x\right\|_{Y_{m}} \rightarrow 0$ as $n \rightarrow \infty$ and the sequence $\left\{\delta_{n}^{K} x\right\}$ is null.

We note in passing that the above result depends on the compactness of $K$. It is well known that if $\int_{-1}^{1} \int_{-1}^{1} w_{2}(t) w_{1}(\tau) k^{2}(t, \tau) d \tau d t$ exists then $K$ is also a compact operator on $H_{1}$ into $H_{2}$. This suggests that the condition that $k$ be Hölder continuous in each variable on $[-1,1]$ can be relaxed.

(vi) $\left\{q_{m} K_{n}\right\}$ is collectively compact on $X_{n}$ into $\operatorname{ran}(A)$. This follows immediately from a result of $\operatorname{Linz}$ [15; Theorem 6] who showed that if $\left\{K_{n}\right\}$ is consistent with a compact operator $K$ and if $\lim _{n \rightarrow \infty}\left\|s_{m} K p_{n}-K_{n}\right\|=0$, then $\left\{q_{m} K_{n}\right\}$ is collectively compact. From (2.13) we have $K_{n}=s_{m} K p_{n}$ so that the result now follows trivially. 
(vii) $\left\{y_{m}-s_{m} y\right\}$ is null. Again, this follows trivially since we have chosen $y_{m}=s_{m} y$.

Thus, for the Galerkin-Petrov method, we have shown that all the conditions of Theorem 5.5 of [2] are satisfied so that convergence of the solutions of the discretized equations to that of the original equation is assured.

Let us conclude this section by considering the rate of convergence. From an equation on page 551 of [2] we have

$$
\left\|r_{n} \psi-x_{n}\right\|_{X_{n}} \leqslant C_{2}\left\{\left\|\delta_{n}^{A} \psi\right\|_{Y_{m}}+\left\|\delta_{n}^{K} \psi\right\|_{Y_{m}}+\left\|y_{m}-s_{m} y\right\|_{Y_{m}}\right\}
$$

so that, since $\left\|\delta_{n}^{A} \psi\right\|_{Y_{m}}=0, y_{m}=s_{m} y$ we have simply that

$$
\left\|r_{n} \psi-x_{n}\right\|_{X_{n}} \leqslant C_{2}\left\|K\left(I-P_{n}\right) \psi\right\|_{H_{2}} .
$$

For the Galerkin-Petrov method it is perhaps more appropriate to consider an upper bound for $\left\|\psi-p_{n} x_{n}\right\|_{H_{1}}$. We have

$$
\begin{aligned}
\left\|\psi-p_{n} x_{n}\right\|_{H_{1}} & \leqslant\left\|\psi-p_{n} r_{n} \psi\right\|_{H_{1}}+\left\|p_{n} r_{n} \psi-p_{n} x_{n}\right\|_{H_{1}} \\
& \leqslant\left\|\left(I-P_{n}\right) \psi\right\|_{H_{1}}+C_{2}\left\|K\left(I-P_{n}\right)\right\| \cdot\left\|\left(I-P_{n}\right) \psi\right\|_{H_{1}}
\end{aligned}
$$

from (3.5), since $\left\|p_{n}\right\|=1$ and $\left(I-P_{n}\right)^{2}=I-P_{n}$. Thus we may write

$$
\left\|\psi-p_{n} x_{n}\right\|_{H_{1}} \leqslant\left\{1+C_{2} \alpha_{n}\right\}\left\|\left(I-P_{n}\right) \psi\right\|_{H_{1}},
$$

where $\alpha_{n}=\left\|K\left(I-P_{n}\right)\right\|$ and, as we have observed previously, tends to zero as $n \rightarrow \infty$. This is a standard form of error bound for Galerkin type methods. Since (1.1) is equivalent to (4.1), it follows that if both $f$ and $k$, with respect to its first independent variable, possess continuous derivatives of order $r$ on $[-1,1]$ then $\psi$ possesses a continuous derivative of order $r$ on $[-1,1]$. By a standard argument it then follows that $\left\|\psi-p_{n} x_{n}\right\|_{H_{1}} \leqslant C_{3} n^{-r} \omega_{r}(1 /(n-r))$ for all $n>r$, where $\omega_{r}$ is the modulus of continuity of $\psi^{(r)}$.

\section{Some comments on the Galerkin-Petrov method}

In this section we shall comment on three aspects of the method. Firstly, we shall consider the so-called "direct" and "indirect" methods (see [9], [10]) for singular integral equations; secondly, we shall look at an "iterated" solution; and finally, we shall consider a particular choice of quadrature rules to evaluate the inner products arising in this method, thereby giving rise to a discretized Galerkin-Petrov method.

For direct methods of solving singular integral equations one discretizes the original equation $(A+K) \psi=y$ as it stands. For indirect methods we consider 
the equivalent Fredholm equation which, if we use the Carleman-Vekua procedure (see [17]), is given by

$$
\left(I+\hat{A}^{I} K\right) \psi=\hat{A}^{I} y+\psi^{(0)},
$$

where $\psi^{(0)}$ is any solution of $A \psi=0$, and find an approximate solution to this equation. We can look upon (4.1) as an operator equation on $H_{1}$ into itself. If we apply the Bubnov-Galerkin method (see [13]) directly to (4.1) we obtain an approximate solution $\psi_{n}^{F}$ say, such that $\psi_{n}^{F}=P_{n} \psi_{n}^{F}$ and satisfies

$$
\psi_{n}^{F}+P_{n} \hat{A}^{I} K \psi_{n}^{F}=P_{n} \hat{A}^{I} y+\psi^{(0)},
$$

where we assume $n$ is chosen large enough so that $\psi^{(0)}=P_{n} \psi^{(0)}$. On the other hand, with the direct method we obtain the algebraic equations (2.4) which are equivalent to

$$
x_{n}+\hat{A}_{m}^{I} K_{n} x_{n}=\hat{A}_{m}^{I} y_{m}+x_{n}^{(0)},
$$

where $A_{n} x_{n}^{(0)}=0$. From (4.3) we compute the vectors $x_{n}$ which give rise to solutions $\psi_{n}^{G}$ say, where $\psi_{n}^{G}=p_{n} x_{n}$ and satisfies

$$
\psi_{n}^{G}+P_{n} \hat{A}^{I} Q_{m} K \psi_{n}^{G}=P_{n} \hat{A}^{I} Q_{m} y+\psi^{(0)},
$$

where we have used (2.13) and (2.15), and also chosen the same solution $\psi^{(0)}$ as we had in (4.2). The question arises as to whether $\psi_{n}^{F}$ and $\psi_{n}^{G}$ are the same. That this is so follows immediately by observing that for any $y \in H_{2}$ and $n$ large enough we have

$$
\begin{aligned}
P_{n} \hat{A}^{I} Q_{m} y & =\hat{A}^{I} Q_{m}^{2} y, \quad \text { by }(2.14), \\
& =\hat{A}^{I} Q_{m} y, \quad \text { since } Q_{m}^{2}=Q_{m} .
\end{aligned}
$$

It follows that equations (4.2) and (4.4) are indeed the same so that $\psi_{n}^{G}=\psi_{n}^{F}$. This has been shown previously by Ioakimidis [10] for the particular case of (1.1) when $a, b$ are constants.

Since, as we have already observed, equations (1.4) and (4.1) are equivalent it follows that techniques developed for Fredholm integral equations could well be applied to singular integral equations. In particular we propose now to consider briefly an iterated solution $\psi_{n}^{*}$ which is defined by

$$
\psi_{n}^{*}=\hat{A}^{I} y-\hat{A}^{I} K \psi_{n}^{G}
$$

It is not difficult to show that $\psi_{n}^{*}$ satisfies a Fredholm equation since

$$
\begin{aligned}
\psi_{n}^{*}+\hat{A}^{\prime} K P_{n} \psi_{n}^{*} & =\hat{A}^{I} y-\hat{A}^{\prime} K \psi_{n}^{G}+\hat{A}^{I} K P_{n} \psi_{n}^{*} \\
& =\hat{A}^{\prime} y-\hat{A}^{\prime} K\left\{\psi_{n}^{G}-P_{n}\left(\hat{A}^{I} y-\hat{A}^{I} K \psi_{n}^{G}\right)\right\}, \text { from (4.5), } \\
& =\hat{A}^{\prime} y, \quad \text { from }(4.2),
\end{aligned}
$$


where we recall that $\psi_{n}^{F}=\psi_{n}^{G}$ and where we have neglected $\psi^{(0)}$, there being no need to "improve" on the solution of the homogeneous equations since we have compatibility of our operators $A, A_{n}$. To recapitulate, $\psi_{n}^{*}$ satisfies the Fredholm equation

$$
\psi_{n}^{*}+\hat{A}^{\prime} K P_{n} \psi_{n}^{*}=\hat{A}^{I} y
$$

from which it follows on comparison with (4.5) that $P_{n} \psi_{n}^{*}=\psi_{n}^{G}$, so that $\psi_{n}^{*}$ is not tô ùe founủ in the suob-space $T_{n}$ of $\dddot{H}_{1}$. On eliminating $\hat{A}^{i} y$ between (4.1) and (4.5) we have

$$
\psi-\psi_{n}^{*}=\hat{A}^{I} K\left(\psi_{n}^{G}-\psi\right)
$$

and this enables us to compare $\left\|\psi-\psi_{n}^{*}\right\|_{H_{1}}$ with $\left\|\psi-\psi_{n}^{G}\right\|_{H_{1}}$. Following Sloan [19] we have

$$
\begin{aligned}
\psi-\psi_{n}^{*} & =\left(I+\hat{A}^{I} K P_{n}\right)^{I}\left(I+\hat{A}^{I} K P_{n}\right) \hat{A}^{I} K\left(\psi_{n}^{G}-\psi\right) \\
& =\left(I+\hat{A}^{\prime} K P_{n}\right)^{I}\left\{\hat{A}^{I} K\left(\psi_{n}^{G}-\psi\right)+\hat{A}^{I} K\left(P_{n} \psi-\psi_{n}^{G}\right)\right\}
\end{aligned}
$$

since $P_{n} \hat{A}^{I} K\left(\psi_{n}^{G}-\psi\right)=P_{n} \psi-\psi_{n}^{G}$ from (4.1) and (4.2). Again, since $P_{n} \psi_{n}^{G}=\psi_{n}^{G}$ we find

$$
\psi-\psi_{n}^{*}=\left(I+\hat{A}^{I} K P_{n}\right)^{I}\left(\hat{A}^{I} K-\hat{A}^{I} K P_{n}\right)\left(\psi_{n}^{G}-\psi\right),
$$

from which we obtain

$$
\left\|\psi-\psi_{n}^{*}\right\|_{H_{1}} \leqslant \beta_{n}\left\|\psi-\psi_{n}^{G}\right\|_{H_{1}}
$$

where

$$
\beta_{n}=\left\|\left(I+\hat{A}^{I} K P_{n}\right)^{I}\right\|\left\|\hat{A}^{I} K\left(I-P_{n}\right)\right\| .
$$

Since $\hat{A}^{I}$ is bounded and $K$ is compact, $\hat{A}^{\prime} K$ is compact and it follows that $\beta_{n} \rightarrow 0$ as $n \rightarrow \infty$, so that $\psi_{n}^{*}$ converges more quickly to $\psi$ than does $\psi_{n}{ }^{G}$. This analysis generalizes that given by Fromme and Golberg [7; Chapter 5] in the particular case of (1.1) with $a=0, b=1$ and $\kappa=0$.

Finally in this section we shall consider the approximate solution obtained when the inner products cannot be evaluated exactly and are approximated by particular quadrature sums. As in [3] and [4] we shall let $\tau_{j, n}, j=1(1) n$, denote the zeros of $t_{n}$ and let $\mu_{j, n}$ be the corresponding Christoffel numbers. Furthermore we shall let $t_{i, m}, i=1(1) m$ denote the zeros of $u_{m}$ with $\nu_{t, m}$ being the corresponding Christoffel numbers. Then we have, see (1.9),

$$
\begin{aligned}
& (f, g)_{H_{1}}=\sum_{j=1}^{n} \mu_{j, n} f\left(\tau_{j, n}\right) g\left(\tau_{j, n}\right)+\text { rem., } \\
& (f, g)_{H_{2}}=\sum_{i=1}^{m} \nu_{i, m} f\left(t_{t, m}\right) g\left(t_{i, m}\right)+\text { rem., }
\end{aligned}
$$


where the remainder terms are zero when $f g$ is a polynomial of degree $\leqslant 2 n-1$ in (4.10) and of degree $\leqslant 2 m-1$ in (4.11). From (2.2) and (2.3) we see that $\psi_{n}^{G}$ satisfies the equations

or

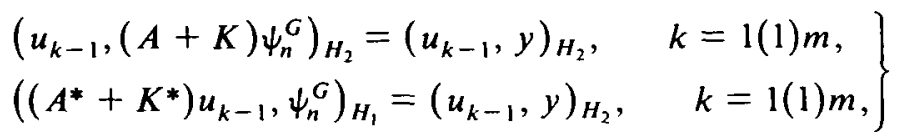

where the adjoint operator $K^{*}$ is defined by

$$
K^{*} \psi(\tau)=\int_{-1}^{1} w_{2}(\xi) k(\xi, \tau) \psi(\xi) d \xi
$$

Let us now replace the inner products in (4.12) by (4.10) and (4.11) with remainder put equal to zero. Again, on using Hunter's quadrature rule for $A^{*} u_{k-1}$ (see, for example, [3]) we find that

$$
A^{*} u_{k-1}\left(\tau_{j, n}\right)=\sum_{i=1}^{m} \frac{\nu_{t, m} u_{k-1}\left(t_{t, m}\right) b\left(t_{t, m}\right)}{\pi\left(\tau_{j, n}-t_{t, m}\right)}
$$

exactly, for $j=1(1) n$. If we replace $K^{*} u_{k-1}\left(\tau_{j, n}\right)$ by

$$
\sum_{j=1}^{m} \nu_{i, m} k\left(t_{t, m}, \tau_{j, n}\right) u_{k-1}\left(t_{t, m}\right)
$$

then from (4.12) on neglecting all the remainder terms we obtain a solution $\tilde{\psi}_{n}^{G}$ say which satisfies the equations

$$
\begin{array}{r}
\sum_{j=1}^{n} \mu_{J, n}\left\{\sum_{i=1}^{m} \nu_{t, m}\left[\frac{u_{k-1}\left(t_{t, m}\right) b\left(t_{t, m}\right)}{\pi\left(\tau_{j, n}-t_{t, m}\right)}+k\left(t_{t, m}, \tau_{j, n}\right) u_{k-1}\left(t_{i, m}\right)\right]\right\} \tilde{\psi}_{n}^{G}\left(\tau_{j, n}\right) \\
=\sum_{i=1}^{m} \nu_{t, m} u_{k-1}\left(t_{i, m}\right) y\left(t_{t, m}\right)
\end{array}
$$

for $k=1(1) m$. We may rearrange this equation to give the system of equations

$$
\begin{array}{r}
\sum_{i=1}^{m} \nu_{t, m} u_{k-1}\left(t_{t, m}\right)\left\{\sum_{j=1}^{n}\left[\frac{b\left(t_{i, m}\right) \mu_{j, n}}{\pi\left(\tau_{j, n}-t_{t, m}\right)}+\mu_{j, n} k\left(t_{t, m}, \tau_{J, n}\right)\right] \tilde{\psi}_{n}^{G}\left(\tau_{j, n}\right)\right. \\
\left.-y\left(t_{t, m}\right)\right\}=0
\end{array}
$$


for $k=1(1) m$. Now for the method of classical collocation it was shown (see Sections 2,6 of [3]) that the approximate solution, which we shall here call $\psi_{n}^{C}$, satisfied the equations

$$
\sum_{J=1}^{n}\left\{\frac{b\left(t_{i, m}\right) \mu_{j, n}}{\pi\left(\tau_{j, n}-t_{t, m}\right)}+\mu_{j, n} k\left(t_{t, m}, \tau_{j, n}\right)\right\} \psi_{n}^{C}\left(\tau_{j, n}\right)=y\left(t_{i, m}\right)
$$

for $i=1(1) \mathrm{m}$. On comparing the systems of equations $(4.15)$ and $(4,16)$ it follows, since the matrix $\left(u_{k-1}\left(t_{i, m}\right)\right)$ is non-singular, that they are equivalent so that for large enough $n$ we have

$$
\tilde{\psi}_{n}^{G}\left(\tau_{j, n}\right)=\psi_{n}^{C}\left(\tau_{j, n}\right), \quad j=1(1) n .
$$

Thus this particular "discretized" Galerkin-Petrov method gives the same solution as the classical collocation method for the complete equation. This generalizes a result of Fromme and Golberg [7; Chapter 4] for the particular case of (1.1) with $a=0, b=1$ and $\kappa=0$.

\section{Conclusion}

We have described in this paper the analysis of a Galerkin-Petrov method for the complete singular integral equation given by (1.1), the only restriction on this equation being that we have assumed throughout that $b$ is a polynomial of degree $\mu$. The analysis given here generalizes that of many authors who have considered only particular cases of (1.1). Furthermore, it demonstrates the applicability, in another context, of the author's general convergence theorem for singular integral equations given in [2]. This analysis also points out the importance of the relationship $A P_{n}=Q_{m} A$, given by Lemma 2.1 from which many of the conditions required for the convergence theorem follow in a particularly simple and elegant way. It was also important in Section 4 for showing the equivalence of certain direct and indirect methods of approximate solution. Considerable work remains to be done on the implementation of this Galerkin-Petrov method and it is hoped to make this the topic of a future paper.

\section{References}

[1] A. V Dzhishkariani, "The solution of singular integral equations by approximate projection methods", U.S S R. Computational Math. and Math. Phys. 19 (1980), 61-74.

[2] David Elliott, "A convergence theorem for singular integral equations", J. Austral. Math. Soc. Ser. B $22(1981), 539-552$.

[3] David Elliott, "The classical collocation method for singular integral equations", SIAM J. Numer Anal 19 (1982), 816-832

[4] David Elliott, "Orthogonal polynomials associated with singular integral equations having a Cauchy kernel". SIAMJ Math Anal. 13 (1982), 1041-1052. 
[5] F. Erdogan and G. D. Gupta, "On the numerical solution of singular integral equations", Quart. Appl. Math. 30 (1972), 525-534.

[6] F. Erdogan, "Approximate solutions of systems of singular integral equations", SIAM J. Appl. Math. 17 (1969), 1041-1051.

[7] Michael A. Golberg (ed.), Solution methods for integral equations: theon and applicattons (Plenum, New York, 1979).

[8] Michael A. Golberg, "Galerkin's method for operator equations with non-negative index-with application to Cauchy singular integral equations", J. Math. Anal. Appl (to be published).

[9] N. I. Ioakimidis and P. S. Theocaris, "A comparison between the direct and the classical numerical methods for the solution of Cauchy type singular integral equations", SIAM J. Numer. Anal. 17 (1980), 115-118.

[10] N. I. Ioakimidis, "On the weighted Galerkin method of numerical solution of Cauchy type singular integral equations", SIAM J, Numer. Anal. 18 (1981), 1120-1127.

[11] P. Junghanns and B. Silbermann, "Zur Theorie der Näherungsverfahren für sınguläre Integralgleichungen auf Intervallen", Math. Nachr. 103 (1981), 199-244.

[12] L. N. Karpenko, "Approximate solution of a singular integral equation by means of Jacobi polynomials", J. Appl. Math. Mech. 30 (1966), 668-675.

[13] M. A. Krasnosel'skii, G. M. Vainikko, P. P. Zabreiko, Ya. B. Rutitskii and V. Ya. Stetsenko, Approxımate solution of operator equations (Wolters-Noordhoff, Gröningen, 1972).

[14] Steen Krenk, "On the use of the interpolation polynomial for solutions of singular integral equations", Quart. Appl. Math. 32 (1975), 479-484.

[15] Peter Linz, "A general theory for the approximate solution of operator equations of the second kind", SIAM J. Numer. Anal. 14 (1977), 543-554.

[16] Peter Linz, "An analysis of a method for solving singular integral equations", BIT 17 (1977). 329-337.

[17] N. I. Muskhelishvili, Singular integral equations (P. Noordhoff, Gröningen, 1953).

[18] Ben Noble, "Error analysis of collocation methods for solving Fredholm integral equations", in Topics in numerical analysis (ed. J. J. H. Miller), (Academic Press, New York, 1973), 211-232.

[19] Ian H. Sloan, "Improvement by iteration for compact operator equations", Math. Comp. 30 (1976), 758-764.

[20] K. S. Thomas, "On the approximate solution of operator equations", Numer. Math 23 (1975), 231-239.

[21] K. S. Thomas, "Galerkin methods for singular integral equations", Math. Comp. 36 (1981), 193-205.

[22] F. G. Tricomi, Integral equations (Interscience, New York, 1957). 